\title{
Política educativa en Chile para eliminar las lógicas neoliberales del mercado escolar
}

\section{Política educacional no Chile para eliminar a lógica neoliberal do mercado escolar}

\section{Education policy in Chile to eliminate neoliberal logic in the school market}

\author{
Geo Saura* \\ Hernán Mateluna**
}

\begin{abstract}
RESUMEN
El presente artículo de investigación analiza el intento de eliminar las lógicas de mercado del sistema educativo chileno. En 2015, Chile, uno de los países más privatizados del mundo, trata de eliminar las consecuencias de las lógicas neoliberales de mercado en educación (lucro educativo y selección escolar) a través de la Ley de Inclusión Escolar. Este intento de eliminar las dinámicas de mercado en el sistema educativo es un proceso de ruptura a dos trayectorias: a la trayectoria histórica del sistema educativo chileno y a la trayectoria global de las políticas educativas pro-privatización. El proceso metodológico de esta investigación utiliza elementos analíticos del enfoque de la adopción de la política de la economía política cultural (variación, selección y retención). Los movimientos sociales de protesta de 2006 y 2011 son los procesos para exigir una nueva reforma educativa en la fase de variación. Las políticas educativas basadas en evidencias y la teoría del derecho social se utilizan para justificar las demandas de las protestas sociales en la fase de selección. En la fase de retención, las negociaciones dentro del gobierno de coalición y la influencia de la escuela privada producen giros importantes en los objetivos de la política educativa analizada para eliminar la lógica de mercado del sistema educativo chileno. Como se ha demostrado
\end{abstract}

*Universidad de Granada. Granada, Andalucía, España. E-mail: geosaura@ugr.es - https:// orcid.org/0000-0001-5049-1483

** Universidad Autónoma de Barcelona. Cerdanyola del Vallès, Barcelona, España. E-mail: hernanmateluna@gmail.com - https://orcid.org/0000-0001-8259-4014 
en los resultados y conclusiones, la lógica de mercado todavía está presente en el sistema educativo chileno.

Palabras clave: Política educativa. Mercado escolar. Neoliberalismo. Privatización. Chile.

\title{
RESUMO
}

Este artigo de pesquisa analisa a tentativa de eliminar a lógica de mercado do sistema educacional chileno. Em 2015, o Chile, um dos países mais privatizados do mundo, tentou eliminar as consequências da lógica neoliberal do mercado na educação (lucro educacional e seleção escolar) por meio da Lei de Inclusão Escolar. Essa tentativa de eliminar a dinâmica do mercado no sistema educacional é um processo de ruptura em duas trajetórias: a trajetória histórica do sistema educacional chileno e a trajetória global das políticas educacionais pró-privatização. O processo metodológico desta pesquisa usa elementos analíticos da abordagem de formulação de políticas da economia política cultural (variação, seleção e retenção). Os movimentos de protesto social de 2006 e 2011 são os processos de reivindicação de uma nova reforma educacional na fase de variação. Políticas educacionais baseadas em evidências e teoria do direito social são utilizadas para justificar as demandas de protestos sociais na fase de seleção. Na fase de retenção, as negociações dentro do governo de coalizão e a influência da escola privada produzem mudanças importantes nos objetivos da política educacional analisada para eliminar a lógica de mercado do sistema educacional chileno. Conforme demonstrado nos resultados e conclusões, a lógica do mercado ainda está presente no sistema educacional chileno.

Palavras-chave: Política educacional. Mercado escolar. Neoliberalismo. Privatização. Chile.

\begin{abstract}
This article analyzes the attempt to eliminate market logic of the Chilean education system. In 2015, Chile, one of the most privatized countries in the world, tries to eliminate consequences of neoliberal dynamics (school profit and student selection) through the Law of School Inclusion. This attempt to eliminate market dynamics in the educational system is a process of rupture of two paths: the trajectory of the history of the Chilean education system and the global trajectory of pro-privatization educational policies. The methodological process of this research uses analytical elements of the approach of the adoption of the politics of the cultural political economy (variation, selection and retention). The social movements of protest of 2006 and 2011 are the processes to demand a new educational reform in the
\end{abstract}


variation phase. Evidence-Based Education Policies and social rights theory are used to justify the demands of social protests in the selection phase. The negotiations of coalition government and the influence of the private school produce turns in the objectives of the analyzed educational policy to eliminate the market logic of the Chilean education system in the retention phase. Overall, the results and conclusions show that market dynamics are still present in the Chilean education system.

Keywords: Education policy. School market. Neoliberalism. Privatization. Chile.

\section{Introducción}

"El neoliberalismo nace y muere en Chile". Así rezaba el grafiti en la rebautizada Plaza de la Dignidad durante los primeros días de las multitudinarias protestas sociales en las principales ciudades de Chile entre 2019-2020. Los primeros análisis sobre estas protestas las han comprendido como "Revolución Social", "Estallido social", "Protestas chilenas 2019-2020", "Protestas del Octubre Chileno" o "Revolución de los 30 pesos" (ARAUJO, 2019; SEHNBRUCH; DONOSO, 2020; SOTO; FARDELLA, 2019). Estas multitudinarias protestas sociales las comenzaron los estudiantes del Instituto Nacional por el alza de treinta pesos en el precio del pasaje de metro en la ciudad de Santiago de Chile. Hasta antes de la crisis sanitaria seguían presentes de forma muy activa en las calles chilenas, ya que, el alza de la subida del metro fue, solamente, el detonante para que se activaran un nuevo ciclo de protestas sociales contras las desigualdades socio-económicas del contexto neoliberal chileno. Estas protestas iniciadas por los estudiantes nacen como respuestas a "la instalación del modelo económico neoliberal al que se le adosó un nuevo modelo de sociedad" (ARAUJO, 2019, p. 17). La perdurabilidad de las protestas durante estos meses se ha materializado como procesos de resistencia a los efectos del modelo neoliberal que llevan transformando la sociedad y las subjetividades desde los últimos cuarenta años. De ahí, que las protestas focalicen de modo prioritario la transformación del sistema educativo, por considerar la educación como uno de los principales elementos que ha legitimado las desigualdades socio-económicas del país durante las últimas décadas. Estas protestas sociales iniciadas por estudiantes son encuadradas como el episodio más reciente del resurgir de los procesos de resistencias colectivas previos, como el del movimiento estudiantil de los pingüinos del año 2006 (DONOSO, 2013) y las posteriores del movimiento estudiantil de 2011 
(BELLEI; CABALIN; ORELLANA, 2014). Tanto las protestas actuales como los episodios más relevantes que las anteceden - año 2006 y año 2011 - forman parte de los procesos de resistencia colectivas que están intentando paralizar la extensión de las dinámicas pro-privatización (FALABELLA, 2015) que conforman lo que ha sido conocido como el "experimento neoliberal chileno en educación" de las últimas cuatro décadas (BELLEI, 2015). Aunque las protestas y las movilizaciones sociales para transformar el sistema educativo chileno continúan, a nivel institucional, desde el año 2015, la política educativa chilena está viviendo un proceso de transformaciones que tiene como meta desmercantilizar las lógicas escolares a través de la Ley de Inclusión Escolar $\mathrm{N}^{\mathrm{o}}$ 20.845 del gobierno de Bachelet (BELLEI, 2016; SISTO, 2019).

La reforma de la Ley de Inclusión nace para intentar des-mercantilizar el sistema educativo mediante la eliminación del lucro escolar, la selección escolar y el financiamiento compartido o copago de la educación financiada por el Estado. Este intento de des-mercantilización en Chile para fortalecer el sistema educativo público y regular la educación privada subvencionada es un proceso de ruptura a dos trayectorias. Por un lado, es una ruptura a la trayectoria histórica a la mercantilización educativa del propio contexto chileno. Esto se debe a que el desarrollo de las políticas educativas en Chile, desde la dictadura de Pinochet, y más concretamente desde la reforma de 1980, se ha basado en una expansión de las lógicas de mercado escolar propias de las prácticas neoliberales. Estas lógicas de puesta en práctica de políticas neoliberales han sido ampliamente analizadas desde diferentes investigaciones de relevancia del contexto chileno - ver, por ejemplo: Budnik et al. (2011); Bellei (2015); Corvalán (2013); Falabella (2014); Mizala (2007); Oliva (2010); Ruiz (2010); Santa Cruz (2016). Por otro lado, este intento de desmercantilizar el sistema educativo chileno mediante la Ley de Inclusión es una ruptura a la trayectoria global de las políticas pro-privatización de los sistemas educativos que llevan viviendo la mayoría de países desde las dos últimas décadas (BALL; YOUDELL, 2008; BURCH, 2009; RIZVI; LINGARD, 2010; SHALBERG, 2011). La nomenclatura “dinámicas endógenas de privatización en la educación” y “dinámicas exógenas de privatización de la educación" de Ball y Youdell (2008) han sido útiles para analizar los procesos heterogéneos pro-privatización del movimiento de reformas educativas de mercado globales - ver algunos de los primeros análisis coordinados en diferentes regiones: Gunter et al. (2016); Luengo Navas et al. (2012); Narodowski y Martínez Boom (2016); Peroni (2013). Por eso este intento de ruptura a dos trayectorias de las dinámicas de mercado en educación (histórica y global) es de gran interés analizar las causas y logros de la presente reforma chilena. Además, el análisis de esta política des-mercantilizadora es de interés por ser Chile conocido uno de los sistemas educativos que se ha entendido 
como un modelo pionero de políticas neoliberales aplicadas a la educación en el mundo, así como por estar ante una reforma pionera en el intento de lograr políticas des-mercantilizadoras de la educación (BELLEI, 2016). El objetivo de esta investigación es analizar algunos aspectos del proceso de construcción de la Ley de Inclusión Escolar a través de su trayectoria política (conflictos sociales que propiciaron el surgimiento, fundamentos teóricos de la reforma y disputas políticas para lograr la aprobación) para “des-mercantilizar" el sistema educativo chileno.

\section{Metodología}

Para analizar el proceso de producción de una política que ha sido definida como "des-mercantilizadora", el enfoque elegido se sustenta en la utilización de elementos propios de principios analíticos de la adopción de políticas de la economía política cultural de Jessop (2010). Este enfoque es útil para analizar la adopción de políticas educativas actuales que están produciendo lógicas pro-privatización de los sistemas educativos (ROBERTSON; DALE, 2015; VERGER; FONTDEVILA; ZANCAJO, 2017). Los fenómenos políticos de mercantilización y privatización de la educación, si bien son procesos globales, responden a contextos particulares y se desarrollan mediante una variedad de políticas y trayectorias diversas en los diferentes países que las adoptan (VERGER; ZANCAJO; FONTDEVILA, 2016). En el caso de las políticas que buscan reducir la presencia de estas lógicas de mercado, si bien es un fenómeno nuevo, creemos también que su proceso de institucionalización puede responder a términos similares a los de la privatización en tanto pretenden un cambio institucional a nivel educativo local. Por ello, consideramos que el enfoque de la economía política cultura (JESSOP, 2010) nos permite aproximarnos a esta transformación desde tres aspectos principales. En primer lugar, nos ayuda a entender el proceso de adopción de políticas educativas a partir de prácticas y contextos concretos siempre teniendo en cuenta las dinámicas de las reformas globales. En segundo lugar, las herramientas de Jessop (2010) posibilitan el análisis de estas transformaciones no solamente desde el ámbito económico y político, sino que también desde la dimensión cultural de las ideas y discursos. Esto actualmente es fundamental en las transformaciones políticas en el ámbito educativo, puesto que las ideas intervienen crucialmente en estos procesos proporcionando razones para que los policy makers acepten determinadas soluciones políticas y descarten otras. En tercer lugar, en toda transformación 
política los factores económicos, políticos y culturales interactúan a través de tres mecanismos: variación, selección y retención (JESSOP, 2010). La variación corresponde al momento en que los discursos oficiales sobre políticas educativas entran en crisis y se hace necesaria su revisión. La selección es el momento en que los policy makers elijen determinadas soluciones políticas que se consideran mas adecuadas para solucionar la crisis. La retención constituye a la fase de negociación para que las políticas educativas sean institucionalizadas en los marcos legislativos vigentes del contexto. Estos tres mecanismos permiten a nuestro análisis identificar, clarificar y estructurar lógicamente los diversos factores que experimenta la adopción de una política que tiene por objetivo realizar un cambio "paradigmático" en la educación chilena para la desmercantilización del sistema educativo. Para analizar el proceso de producción de la política, la estrategia de investigación se organiza en tres fases. En primer lugar, se revisa la dimensión teórica-ideológica que fundamenta la reforma educativa desde la perspectiva del derecho social. En segundo lugar, se revisan los artículos de investigación realizados por académicos y que permiten al gobierno justificar las medidas que buscan reducir la presencia del mercado en el sistema educativo chileno desde una manera práctica. En tercer lugar, mediante el análisis documental de las políticas se examinan documentos oficiales relacionados con el proceso de elaboración de la ley (Programa de gobierno, Mensaje presidencial 131-362, Historia de la ley 20.825, Cuenta pública del Ministerio de Educación año 2015, Ley 20.825, Ley General de Educación y leyes que modifican a la ley de inclusión - CHILE, 2015). La examinación de estos documentos se realiza tomando como patrones preestablecidos conceptos claves del proceso de elaboración de la política (lucro, selección escolar, copago, des-mercantilización, mercado escolar, neoliberalismo, política educativa, privatización). En total se revisan cerca de 3.000 páginas de documentos oficiales junto a la literatura científica revisada que han centrado la atención en la política educativa neoliberal del contexto chileno. Los análisis de las políticas y literatura científica se sistematizan con el software NVivo 12 a través de diversos nodos que conforman tres categorías amplias relacionadas con las medidas de la ley: fin al lucro; fin a la selección; y fin al copago.

\section{Variación. Movimientos sociales y lógicas de mercado en educación}

La primera fase de las adopciones políticas hace referencia a la variación, como momento histórico en el que son revisadas las prácticas políticas por 
procesos críticos y movilizaciones sociales que hacen emerger nuevos discursos políticos para intentar ofrecer respuestas a las nuevas demandas. El movimiento estudiantil del año 2006 (DONOSO, 2013) y el movimiento estudiantil del año 2011 (BELLEI, CABALIN; ORELLANA, 2014; GARCÍA-HUIDOBRO, 2011) fueron los ciclos de resistencias colectivas que fomentaron la variación de las políticas para promover el intento des-mercantilizador de la ley de Inclusión (BELLEI, 2016; PICAZO; PIERRE, 2016). Ambos ciclos de resistencia colectiva se centraron en los efectos de las desigualdades sociales de la mercantilización expansiva del sistema educativo chileno y en demandar mayor participación estatal en la provisión educativa. Esto se debe a que ambos procesos de resistencia compartieron en su discurso el descontento ante los efectos que tienen las lógicas de mercado en el sistema educativo, por lo que exigen un cambio estructural en la cual el Estado tenga mayor participación en la provisión de educación. El movimiento de protestas colectivas de 2006, conocido como las resistencias de escolares del "movimiento pingüino", ha sido estudiado como la primera gran expresión de protestas hacia la mercantilización desde el retorno a la democracia - ver: Bellei y Cabalin (2012); Cabalin (2014); Donoso (2013). Estas protestas tenían una visión estratégica para instalar sus demandas contra la estructura del sistema educativo que incluía una agenda corta y una agenda larga (GARRETÓN et al., 2011). La agenda corta se basaba en medidas puntuales para obtener soluciones a corto plazo y la agenda larga con cambios estructurales en la lógica neoliberal del sistema educativo las cuales no se podían obtener de modo inmediato. Las demandas iniciales de la agenda corta de estas protestas comenzaron reclamando la modificación de la jornada escolar completa y la participación de los Centros de Alumnos. Si bien las protestas comenzaron desde los estudiantes secundarios, se fueron estableciendo alianzas más amplias con el profesorado de secundaria y estudiantes universitarios para tener mayor amplitud en las protestas. De estas alianzas para actuar en agenda larga centrada cambios más profundos como en la exigencia para que el Estado abandonara el rol subsidiario. Dentro de las demandas específicas de la agenda larga se priorizó en reformar la ley general de educación LOCE bajo demandas concretas como la gratuidad educativa, el fortalecimiento del sistema educativo público, el rechazo al lucro y poder acabar con la selección de estudiantes (BELLEI; CABALIN, 2013). Aunque las protestas impactaron y modificaron algunos aspectos de la política educativa (BELLEI; CABALIN, 2013), los cambios conseguidos por el movimiento de 2006 que incluye la derogación de la LOCE, no llegan a modificar las lógicas neoliberales del sistema educativo (GARRETÓN et al., 2011). Como se ha manifestado, los modos de conducir el conflicto por parte de los partidos políticos fueron el detonante para consolidar el "Estado evaluador" en el sistema educativo (SANTA CRUZ, 2016; PACERISA; FALABELLA, 2017). 
Para proseguir las resistencias colectivas del movimiento pingüinos sobre la defensa de un sistema educativo público, gratuito y abolir el lucro en educación, en el año 2011 nacen movilizaciones estudiantiles de universitarios (GARCÍA-HUIDOBRO, 2011) que representan mayor madurez y organización respecto al proceso de años anteriores cuando eran estudiantes secundarios (BELLEI; CABALIN; ORELLANA, 2014). Al reconocer que las demandas de las protestas no son procesables con el actual modelo de mercado en Chile, como sostiene uno de los principales dirigentes estudiantiles (BORIC, 2012), el movimiento se centra en intentar superar la "camisa de fuerza pinochetista" con un "cambio de paradigma". La respuesta del gobierno de Piñera y la centroderecha a estas protestas de 2011 pasó por un ajuste del modelo con la inyección de más recursos para educación y mayor regulación sin modificar la estructura del sistema educativo (PICAZO; PIERRE, 2016). De ahí que esta generación de estudiantes nacidos o crecidos en periodo democrático, no se conformaron con esta "política de consensos" que condicionó los cambios más relevantes en el retorno al sistema democrático (MOULIAN, 1997). Como se ha defendido, las ideas neoliberales hegemónicas en la educación chilena no son de "sentido común" (CABALIN, 2012) por lo que este movimiento de protestas colectivas exige cambios estructurales al modelo de mercado en la educación. De ahí que cuatro lideres estudiantiles del movimiento de protestas de 2011 llegaron en el año 2014 al parlamento conformando la "bancada estudiantil" y participando activamente en la ley de Inclusión.

\section{Selección. De la educación de mercado al derecho social}

La segunda fase del proceso de las adopciones políticas es la selección. Tras interpretar los procesos de crisis, los policy makers optan por determinadas soluciones y alternativas políticas que se consideran más adecuadas para solventar los procesos de crisis, a la vez que descartan otras. La ausencia de respuestas a las protestas estudiantiles se presentaba como una oportunidad para que la coalición de centro-izquierda recuperara el gobierno en las elecciones de 2014. El movimiento estudiantil significaba una oportunidad para que esta coalición redefiniera sus discursos apropiándose de las demandas discursivas del movimiento social (SEGOVIA; GAMBOA, 2012) y poder incluirlas dentro del programa de gobierno de la candidata a la presidencia "Bachelet" (BELLEI, 2016). En esta fase de selección (JESSOP, 2010) juegan un rol determinante las ideologías, las interpretaciones discursivas y los paradigmas políticos. El 
factor ideológico más significativo en esta reforma fue la noción del "derecho social" que Atria (2014) reactualiza al presente chileno desde la noción de Marshall (1950). Fernando Atria encuentra en la noción de derecho social un medio para superar aspectos de la desigualdad generadas por las lógicas mercantiles y así revertir la capacidad de pago como medio de obtención de mayor calidad educativa. Atria (2014) establece el "derecho social", como nuevo paradigma para reemplazar el modelo neoliberal. Estos principios ideológicos son defendidos mediante la exigencia a las iniciativas privadas de la eliminación del lucro, de la selección de estudiantes y de la financiación compartida.

Para defender la eliminación de estos tres elementos de la educación subvencionada por el Estado, el gobierno de Bachelet enmarcó la conveniencia bajo los estudios propios de la política educativa basada en evidencia científica. La aspiración de terminar con el lucro en el sistema escolar y de prohibir la selección de estudiantes ya se había intentado introducir sin éxito en el proceso de modificación de la Ley de Educación en el año 2008 (SANTA CRUZ, 2016), por lo que se hizo necesario una nueva estrategia para poder llevar a cabo estos cambios. La eliminación del lucro se justificó con estudios que atribuyen a las escuelas privadas subvencionadas responsabilidad en la segregación del sistema educativo (CONTRERAS et al., 2011) y a través de las evidencias de que las escuelas privadas subvencionadas sin fines de lucro obtienen mejores resultados que las escuelas con fines de lucro en pruebas estandarizadas (CHUMACERO y PAREDES, 2008; ELACQUA, 2009; ZUBIZARRETA, PAREDES y ROSENBAUM, 2014). El fin de la selección se justificó con las evidencias que señalan que la selección es mayor en las escuelas privadas subvencionadas que en las públicas, siendo un factor de segregación al generar escuelas más homogéneas (CARRASCO et al., 2014), a la vez que estas escuelas obtienen mejores resultados en pruebas estandarizadas sin ser mas eficientes en el proceso de enseñanza (CONTRERAS; SEPÚLVEDA; BUSTOS, 2010; HSIEH; URQUIOLA, 2006).

El copago se considera como un factor contribuyente a la segregación socioeconómica del sistema educativo debido a que las escuelas con copago tienen menos porcentaje de estudiantes vulnerables respecto a las gratuitas (ELACQUA, 2012; ELACQUA; MONTT; SANTOS, 2013; VALENZUELA; BELLEI; DE LOS RÍOS, 2014). Si bien las escuelas con copago obtienen mejores resultados que las escuelas gratuitas, al controlar el nivel socioeconómico de la escuela la diferencia se reduce notablemente (ELACQUA; MONTT; SANTOS, 2013; MIZALA; TORCHE, 2012). En la fase de selección de la ley de Inclusión, a diferencia de la tramitación de los cambios a la política educativa del primer gobierno de Bachelet posterior al movimiento del 2006, se utilizaron todos estos estudios para evidenciar la necesidad de eliminar las prácticas de mercado como 
el lucro, la selección y el copago. Desde el Ministerio de Educación (UBEIRA, 2017) se realiza un informe sobre la evidencia académica para demostrar las investigaciones que han evidenciado resultados de los efectos no deseados de la segregación escolar para argumentar en contra de los procesos de selección escolar, fin al lucro y al copago. Los informes y las investigaciones utilizadas forman parte de la utilización de la corriente de las políticas educativas basadas en evidencias que llevan utilizándose para legitimar las políticas chilenas durante las últimas décadas (EGIDO, 2019). Estas políticas educativas basadas en evidencias ahora se normalizan como generadoras de verdades. Estas lógicas de argumentar mediante estudios de políticas educativas basadas en evidencias se han vuelto hegemónicas en los procesos de las reformas educativas globales. De este modo, tanto los estudios empíricos como el pensamiento del derecho social actúan bajo una red de expertos que permiten construir un marco teórico argumentativo para abolir las practicas de mercado en el sistema chileno.

Por su parte, la oposición política no argumentó sus ideas a través de estudios académicos para avalar el lucro, la selección y el copago como medios para mejorar la calidad del sistema educativo. Los discursos de la oposición estaban vacíos de contenidos. La oposición sostenía argumentos como estar ante "una reforma ideológica, de ideología de izquierda, que iguala lo público con lo estatal" (BIBLIOTECA DEL CONGRESO NACIONAL DE CHILE, 2015, p. 809). En la fase de selección también se suelen utilizar las influencias internacionales para justificar las políticas. La oposición no utilizó en defensa otros países que fueran utilizados como modelos para poder argumentar a favor de las lógicas de mercado imperantes de la educación chilena. En las discusiones parlamentarias revisadas que se exponen a continuación, en reiteradas ocasiones los promotores de la ley establecen que Chile es un país paradigmático a nivel mundial en cuanto al lucro y selección escolar con fondos públicos. Estas lógicas de mercado en Chile han sido manifestadas históricamente como una "sociedad de referencia" (SCHRIEWER, 1990) en materia de mercado educativo a nivel internacional.

\section{Retención. Des-mercantilización en la medida de lo posible}

La tercera fase de la adopción de políticas hace referencia a la retención. En esta fase, es donde entran en juego los procesos de negociaciones parlamentarias que hacen que las políticas educativas puedan ser institucionalizadas según marcos nacionales e internacionales. Esta es la fase más compleja, ya que los 
movimientos sociales y las negociaciones entre los diversos grupos políticos pueden trasladar a otro escenario, tanto la política como sus principios ideológicos. En el contexto de la ley de Inclusión, el gobierno, aunque contaba con la mayoría parlamentaria para poder lograr la aprobación y puesta en marcha de la ley, encontró importantes reticencias a la des-mercantilización dentro de su propia coalición, ya que estaba conformada por grupos diversos que van desde el Partido Comunista a partidos más conservadores como la Democracia Cristiana. Las diferencias ideológicas en el gobierno de coalición y la falta de acuerdos previos con los representantes de la oposición hicieron que la ley sufriera cerca de 600 indicaciones para su modificación (BACHELET, 2015).

Los intentos por establecer las transformaciones al sistema educativo de mercado a partir de la eliminación del lucro, selección escolar y financiamiento compartido se enfrentó a una oposición que consideró que estas prohibiciones afectan la libertad de los sostenedores para desarrollar su propio proyecto educativo y de las familias para elegirlos. A este discurso se agregaron las presiones de los dueños de escuelas privadas subvencionadas que consideraron que de no flexibilizar las medidas, cerca de un $70 \%$ de estas escuelas se verían obligadas a cerrar (BIBLIOTECA DEL CONGRESO NACIONAL DE CHILE, 2015, p.100). Estos argumentos presionaron al gobierno, ya que el Estado no podía prescindir de estas escuelas por la proporcionalidad que ocupan en el sistema educacional (BELLEI, 2016).

A las presiones "externas" contra los cambios propuestos por el gobierno, se sumaron internamente las de su propia coalición. En Chile, al igual que comienzan a suceder en otros contextos (por ejemplo, en el estado español), los gobiernos de coalición proceden de ideologías y tradiciones muy diversas, siendo esto algo que complejiza lograr acuerdos de gobierno que tomen medidas tan contundentes como des-mercantilizar las lógicas históricas de la privatización en educación. En ese sentido, dentro de la coalición, el partido de la Democracia Cristiana fue el que mas reticencia mostró a los cambios y presionó para frenar algunas medidas. Las presiones externas como internas obligaron al gobierno a ceder en algunos puntos de lo propuesto en el proyecto de ley, debiendo realizar modificaciones a las medidas originalmente propuestas respecto a la prohibición al lucro, selección escolar y financiamiento compartido.

En relación a la prohibición al lucro, un aspecto central de la ley era evitar el lucro encubierto a través del retiro de utilidades mediante "sociedades espejo" que generan ganancias mediante "autoarriendos" (MÖNCKEBERG, 2007). Para evitar esto, el proyecto de ley obligaba a los dueños de escuelas a ser propietarios de sus establecimientos. Sin embargo, el gobierno flexibiliza estas medidas previa negociación con la Iglesia Católica (LA TERCERA, 2014) y con la propia Democracia Cristiana (BIBLIOTECA DEL CONGRESO NACIONAL 
DE CHILE, 2015, p.995). Así pues, se permite que los dueños de escuelas mantengan sus contratos de arriendo ya celebrados durante un plazo de hasta 8 años, sin considerar si el arrendatario tiene fines de lucro y está relacionado con el arrendador (Ley 20845, 2015, Art. $2^{\circ}$ y $5^{\circ}$ transitorio - CHILE, 2015). Este plazo será nuevamente ampliado con modificaciones realizadas a la ley en el año 2017 (Ley 20.993, 2017 - CHILE, 2017).

Respecto a la selección escolar, el proyecto original prohibía los procesos de selección en todas las escuelas subvencionadas por el Estado. Ante las presiones de los dueños y representantes de escuelas privadas subvencionadas denominadas de alta exigencia (BIBLIOTECA DEL CONGRESO NACIONAL DE CHILE, 2015, p.109), así como de un sector de gobierno que veía amenazada la continuidad de los liceos emblemáticos considerados bastiones de la educación pública (SALLABERRY, 2014), el gobierno incluyó modificaciones para diferenciar la selección arbitraria de la por mérito, para que las escuelas denominadas de alta exigencia pudiesen establecer sus propios procesos de admisión a un $30 \%$ de las vacantes a partir de $7^{\circ}$ año básico (Ley 20845, 2015, numeral 6 del Art. $2^{\circ}$ - CHILE, 2015). La exigencia de no seleccionar estudiantes no incluyó a las escuelas privadas, a pesar de las indicaciones sugeridas por miembros de la "bancada estudiantil", las cuales no fueron aprobadas.

Finamente, la eliminación del financiamiento compartido fue cuestionada por la oposición por no permitir que los padres legítimamente puedan contribuir económicamente a la educación de sus hijos (BIBLIOTECA DEL CONGRESO NACIONAL DE CHILE, 2015, p.395). A este discurso se suman representantes de la Democracia Cristiana que como solución plantean que se autoricen aportes voluntarios y esporádicos que permitan a las comunidades educativas organizar sus actividades extracurriculares (BIBLIOTECA DEL CONGRESO NACIONAL DE CHILE, 2015, p.1189). Esto es finalmente incluido en la ley (Ley 20845, 2015, Art. 20, numeral 5, letra j).

De todo lo anterior se puede dilucidar que el gobierno cedió ante las presiones de los proveedores privados, quienes, en defensa de sus intereses, utilizaron su transversalidad e influencias en el espectro político para minimizar los cambios al modelo educativo. Esto obligó al gobierno a consensuar los cambios mas significativos para poder sacar adelante la ley. En definitiva, estos consensos son los que no han permitido el despliegue suficiente de una ley que busca eliminar la presencia del mercado en educación y, por el contrario, han terminado por consolidar prácticas de mercado en la educación escolar que recibe fondos públicos. 


\section{Conclusiones}

Este artículo ha presentado un análisis de diversos factores que operan en la adopción de la ley de Inclusión como política educativa que intenta eliminar las lógicas de mercado del sistema educativo chileno. La política educativa intenta des-mercantilizar el sistema educativo mediante la eliminación del lucro, la selección escolar y el financiamiento compartido o copago de la educación financiada por el Estado. Este intento de des-mercantilizar el sistema educativo chileno es una ruptura a dos trayectorias. Por un lado, es una ruptura a la trayectoria histórica de la mercantilización educativa que lleva viviendo Chile durante los últimos cuarenta años. Por otro lado, es una ruptura a la trayectoria global de las políticas pro-privatización que están modificando la mayoría de sistemas educativos bajo lógicas de mercado. Para el análisis del proceso de la reforma educativa, se ha optado por ampliar elementos de análisis propios de las adopciones políticas que se suelen utilizar desde la de la economía política cultural. En relación a primera fase de variación, se toma en cuenta las protestas sociales estudiantiles del año 2006 y del año 2011 como ciclos de resistencias colectivas más relevantes como punto de partidas para intentar promover la variación de mercado del sistema educativo chileno. Respecto a la fase de selección de la adopción de la política educativa, se utiliza la noción ideológica del derecho social y estudios académicos basados en evidencias para justificar la necesidad de eliminar prácticas de mercado en el sistema educativo chileno. Para ello, se utilizan evidencias que señalan que la selección escolar, el copago y el lucro educativo normalizado en Chile son factores que promueven la segregación socioeconómica del sistema educativo sin haber mejorado la calidad de la educación en su conjunto.

Respecto a la fase de retención, que es donde entran en juego los procesos de negociaciones políticas, la idea inicial de des-mercantilizar la educación encontró fuertes resistencias dentro de la propia coalición de gobierno para poder desplegar el proyecto original. La oposición política defendió las lógicas mercantiles del modelo educativo fundamentándose en que las medidas afectan las libertades de los distintos actores educativos. Los dueños y representantes de las escuelas privadas subvencionadas presionaron al gobierno para flexibilizar las medidas asegurando que las prohibiciones afectarían el funcionamiento de sus escuelas y los obligaría a cerrar un porcentaje alto de sus establecimientos, perjudicando a estudiantes de clase media que tienen en esta modalidad escolar como única opción.

Los cambios propuestos por esta ley representan un avance en la tradición escolar chilena "en la medida de lo posible" al establecer por primera vez 
condiciones al sector privado para mantener la denominada "igualdad de trato" entre escuelas públicas y privadas, lo que acerca al sistema educativo chileno a prácticas habituales de otros países como Bélgica u Holanda - véase: Bellei (2015). Esta nueva relación que establece el Estado con el ámbito privado puede enmarcarse dentro de las nuevas alianzas público privada expandidas durante las últimas décadas (ADRIÃO; PERONI, 2008), que se actualizan con lógicas en las que los gobiernos guían la política y el financiamiento mientras los privados brindan los servicios educativos (PATRINOS; BARRERA-OSORIO; GUÁQUETA, 2009). Si bien, esto supone un avance a nivel local, no obstante, opera en sintonía con las medidas pro-privatización desde la perspectiva internacional. Asimismo, el intento de superación del neoliberalismo en la educación chilena supone una paradoja, puesto que sus cambios se sustentan en la arquitectura neoliberal impuesta por la dictadura y sus principios del school choice y el financiamiento por vouchers (SISTO, 2019). En ese sentido, el intento chileno pretende des-mercantilizar la educación sin modificar estos principios, y, por el contrario, los consolida. Precisamente, la eliminación de las barreras económicas del copago y de la selección que realizaban las escuelas subvencionadas por el Estado, busca dar libertad real a las familias para la elección de escuela. Por otra parte, el financiamiento por vouchers se consolida con la ampliación de las subvenciones adicionales que entregará el Estado a las escuelas para reemplazar el cobro del copago. En definitiva, esta política no elimina los procesos de mercado, sino que es un nuevo ajuste de la mercantilización educativa en Chile.

\section{REFERENCIAS}

ADRIÃO, Theresa; PERONI, Vera Maria Vidal (org.). Público e privado na educação: novos elementos para o debate. São Paulo: Xamã. 2008.

ARAUJO, Kathya (ed.). Hilos tensados. Para leer el octubre chileno. Santiago: Editorial USACH. 2019.

BUDNIK, Jenny Assaél et al. La empresa educativa chilena. Educaçao \& Sociedade, Campinas, v. 32, n. 115, p. 305-322, abr.-jun. 2011.

ATRIA, Fernando. Derechos sociales y educación: un nuevo paradigma de lo público. Santiago: LOM ediciones, 2014.

BACHELET, Michelle. Discurso de S.E. la Presidenta de la República, Michelle Bachelet, al promulgar Ley n 20.845 de Inclusión Escolar. 2015. Disponible en: http:// 
archivospresidenciales.archivonacional.cl/uploads/r/archivo-presidencia-de-la-republic a/5/3/7/537c5d2c4001e431080f379a3c80e6cb129fc83ebf5474ba904554671cc10674/ home_aristoteles_documentos_DIS_0584.pdf Acceso en : 22 mayo 2020.

BALL, Stephen J.; YOUDELL, Deborah. Hidden privatisation in public education. Brussels: Education International, 2008.

BELLEI, Cristián. El gran experimento: Mercado y privatización de la educación chilena. Santiago de Chile: LOM Ediciones. 2015.

BELLEI, Cristián. Dificultades y resistencias de una reforma para des-mercantilizar la educación. RASE: Revista de La Asociación de Sociología de La Educación, València, v. 9, n. 2, p. 232-247, 2016.

BELLEI, Cristián; CABALÍN, Cristian. Chilean student movements: sustained struggle to transform a market-oriented educational system. Current Issues in Comparative Education, New York, NY, v. 15, n. 2, p. 108-123, 2013.

BELLEI, Cristián; CABALIN, Cristian.; ORELLANA, Víctor. The 2011 Chilean student movement against neoliberal educational policies. Studies in Higher Education, v. 39, n. 3, p. 426-440, 2014.

BIBLIOTECA DEL CONGRESO NACIONAL DE CHILE (BCN). Historia de la Ley $N^{o}$ 20845, Chile: BNC, 2015.

BORIC, Gabriel. Los horizontes del movimiento estudiantil. Le Monde Diplomatique. (Edición Chilena), Santiago, mayo 2012. Disponible en: https:/www.lemondediplomatique. cl/Los-horizontes-del-movimiento.html. Acceso en: 24 mayo 2020

BURCH, Patricia. Hidden markets. The new education privatisation. New York and London: Routledge, 2009.

CABALIN, Cristian. Neoliberal Education and Student Movements in Chile: Inequalities and Malaise. Policy Futures in Education, [s.l.], v. 10, n. 2, p. 219-228, 2012.

CABALIN, Cristian. Estudiantes conectados y movilizados: El uso de Facebook en las protestas estudiantiles en Chile [Online and Mobilized Students: The Use of Facebook in the Chilean Student Protests]. Comunicar, Chile, v. 22, n. 43, p. 25-33, 2014.

CARRASCO, Alejandro et al. Selección de estudiantes y desigualdad educacional en Chile: ¿qué tan coactiva es la regulación que la prohíbe? Informe proyecto FONIDE 711286. Santiago: CEPPE, 2014.

CHILE, República de. Ley No 20.845. De inclusión escolar que regula la admisión de los y las estudiantes, elimina el financiamiento compartido y prohíbe el lucro en establecimientos educacionales que reciben aportes del Estado. Santiago: Diario Oficial de la República de Chile, 2015.

CHILE, República de. Ley No 20.993. Modifica diversos cuerpos legales para facilitar el funcionamiento del sistema escolar. Santiago: Diario Oficial de la República de Chile, 2017. 
CHUMACERO, Romulo; PAREDES Ricardo. Should for profit schools be banned?, MPRA paper, No 15099, University Library of Munich, 2008.

CONTRERAS, Dante et al. El Lucro en la Educación Escolar. Evidencia y desafios regulatorios. Santiago: Departamento de Economía, Universidad de Chile, 2011.

CONTRERAS, Dante; SEPÚLVEDA, Paulina; BUSTOS, Sebastián. When Schools Are the Ones that Choose: The Effects of Screening in Chile. Social Science Quarterly, [s.l.], v. 91, n. 5, p. 1349-1368, 2010.

CORVALÁN, Javier. La narrativa educacional chilena y su proceso de transformación reciente. Folios, Bogotá, v. 37, p. 63-81, 2013.

DONOSO, Sofia. Dynamics of Change in Chile: Explaining the Emergence of the 2006 Pingüino Movement. Journal of Latin American Studies, Cambridge, UK, v. 45, n. 1, p. 1-29, 2013.

EGIDO, Miriam Pietro. Percepciones del profesorado sobre las políticas de aseguramiento de la calidad educativa en chile. Educaçao e Sociedade, Campinas, v. 40, p. 1-20. 2019.

ELACQUA, Gregory. For-Profit Schooling and the Politics of Education Reform in Chile. When Ideology Trumps Evidence. Santiago: Centro de Políticas Comparadas en Educación, 2009.

ELACQUA, Gregory. The impact of school choice and public policy on segregation: Evidence from Chile. International Journal of Educational Development, [s.l.], v. 32, n.3, p. 444-453, 2012.

ELACQUA, Gregory; MONTT, Pedro; SANTOS, Humberto. Evidencias para eliminar -gradualmente- el Financiamiento Compartido. Claves de Politicas Publicas, Chile, v. 14, p. 1-10, mayo 2013.

FALABELLA, Alejandra. The Performing School: Effects of Market \& Accountability Policies. Education Policy Analysis Archives, Arizona, v. 22, n. 70, 2014.

FALABELLA, Alejandra. El mercado escolar en Chile y el surgimiento de la Nueva Gestión Pública: el tejido de la política entre la dictadura neoliberal y los gobiernos de la centroizquierda (1979 a 2009). Educaçao \& Sociedade, Campinas, v. 36, n. 132, p. 699-722, 2015.

GARCÍA-HUIDOBRO, Juan Eduardo. Movilizaciones estudiantiles, un intento de interpretación. Cuaderno de Educación, Santiago, v. 35, p.1-10, 2011.

GARRETÓN, Manuel Antonio et al. Movimiento social, nuevas formas de hacer política y enclaves autoritarios: Los debates del Consejo Asesor para la Educación en el gobierno de Michelle Bachelet en Chile. Polis, Santiago, v. 10, n. 30, p. 117-140, 2011.

GUNTER, Helen M. et al. New Public Management and the Reform of Education: European Lessons for Policy and Practice. London \& New York: Routledge. 2016. 
HSIEH, Chang-Tai; URQUIOLA, Miguel. The effects of generalized school choice on achievement and stratification: Evidence from Chile's voucher program. Journal of Public Economics, [s.l.], v. 90, p. 1477-1503, 2006.

JESSOP, Bob. Cultural political economy and critical policy studies. Critical Policy Studies, Lancaster, UK, v. 3, n. 3-4, p. 336-356, 2010.

LA TERCERA. Mineduc logra acuerdo con la Iglesia sobre arriendo regulado y admisión escolar. La Tercera. 2014. Disponible en: https://www.latercera.com/noticia/mineduclogra-acuerdo-con-la-iglesia-sobre-arriendo-regulado-y-admision-escolar. Acceso el: 24 mayo 2020

LUENGO NAVAS, Juan et al. Nuevas formas, nuevos actores y nuevas dinámicas de la privatización en educación. Profesorado. Revista de curriculum y formación del profesorado, Granada, v. 16, n. 3, p. 3-12, 2012.

MARSHALL, Thomas Humphrey. Citizenship and Social Class and Other Essays. Cambridge: Cambridge University Press, 1950.

MIZALA, Alejandra. La economía política de la reforma educacional en Chile. Serie Estudios Socio/Económicos, Chile, n. 36, p. 1-48, 2007.

MIZALA, Alejandra; TORCHE, Florencia. Bringing the schools back in: The stratification of educational achievement in the Chilean voucher system. International Journal of Educational Development, [s.l.], v. 32, n. 1, p. 132-144, 2012.

MÖNCKEBERG, María Olivia. El negocio de las universidades en Chile. Santiago: Debate, 2007.

MOULIAN, Tomás. Chile anatomía de un mito. Santiago: LOM Ediciones, 1997. 2007.

NARODOWSKI, Mariano; MARTÍNEZ BOOM, Alberto. Presentación. Revista Colombiana De Educación, Bogotá, v.70, p. 13-14, jan./jun. 2016.

OLIVA, María Angélica. Política educativa chilena 1965-2009. ¿Qué oculta esa trama? Revista Brasileira de Educação, Rio de Janeiro, v. 15, n. 44, p. 311-328, 2010.

PARCERISA, Lluís; FALABELLA, Alejandra. La consolidación del Estado evaluador a través de políticas de rendición de cuentas: Trayectoria, producción y tensiones en el sistema educativo chileno. Education Policy Analysis Archives, Arizona, v. 25, n. 89, 2017.

PATRINOS, Harry Anthony; BARRERA-OSORIO, Felipe; GUÁQUETA, Juliana. The role and impact of public-private partnerships in education. Washington D.C.: World Bank Publications, 2009.

PERONI, Vera Maria Vidal (org.). Redefinições das fronteiras entre o público e o privado: implicações para a democratização da educação. Brasília: Liber Livro, 2013.

PICAZO, María Inés; PIERRE, Christelle. La educación como derecho social: La construcción del referencial de acción pública del movimiento estudiantil chileno. Revista Uruguaya de Ciencia Política, Montevideo, v. 25, n. 2, p. 99-120, 2016. 
RIZVI, Fazal; LINGARD, Bob. Globalizing educational policy. London: Routledge. 2010 .

ROBERTSON, Susan L.; DALE, Roger. Towards a "critical cultural political economy" account of the globalising of education. Globalization, Societies and Education, [s.l.], v. 13, n.1, p. 149-170, 2015.

RUIZ, Carlos. De la república al mercado. Santiago: LOM Ediciones, 2010.

SAHLBERG, P. Finnish Lessons: what can the world learn from educational change in Finland? New York: Teachers College Press. 2011.

SALLABERRY, Juan Pablo. ¿Selección Nacional? Revista Qué Pasa, 2014. Disponible en: http:/www.quepasa.cl/articulo/actualidad/2014/05/1-14309-9-seleccion-nacional. shtml/. Acceso en: 23 jul. 2020.

SANTA CRUZ, Eduardo. Mediatización de las politicas educativas en Chile: El discurso de los diarios La Tercera y El Mercurio sobre la Ley General de Educación (2006-2009). 2016. Tesis (Doctoral Ciencias de la Educación) - Faculdad de Ciencia e Educación, Universidad de Granada, Granada. 2016.

SCHRIEWER, Jgen. The Method of Comparison and the Need for Externalization: Methodological Criteria and Sociological Concepts. En: Jgen. Schriewer y Brian. Holmes (ed.) Theories and Methods in Comparative Education. Frankfurt am Main: Peter Lang, 1990. p. 25-83.

SEGOVIA, Carolina; GAMBOA, Ricardo. Chile: el año en que salimos a la calle. Revista de Ciencia Política, Santiago, v. 32, n. 1, p. 65-86, 2012.

SEHNBRUCH, Kirsten; DONOSO, Sofia. Social protests in Chile: inequalities and other inconvenient truths about Latin America's poster child. Global Labour journal, Johannesburg, South Africa, v. 11, n. 1, p. 52-58, 2020.

SISTO, Vicente. Inclusión “a la Chilena”: La inclusión escolar en un contexto de políticas neoliberales avanzadas. Archivos Analíticos de Políticas Educativas, Arizona, v. 27, n. 23, p. 1-20, 2019.

SOTO, Alvaro; FARDELLA, Carla. Del yo al nosotros: el emplazamiento colectivo a las subjetividades neoliberales. En: ARAUJO, Katia (ed.). Hilos tensados. Para leer el octubre chileno. Santiago: Editorial USACH, 2019. p. 243-270.

UBEIRA, Felipe. Evidencia académica en torno a elementos de la Ley de Inclusión: copago y subvenciones, lucro y selección. Ministerio de Educación: Santiago. 2017.

VALENZUELA, Juan Pablo; BELLEI, Cristian; DE LOS RÍOS, Danae. Socioeconomic school segregation in a market-oriented educational system. The case of Chile. Journal of education Policy, [s.l.], v. 29, n. 2, p. 217-241, 2014.

VERGER, Antoni; FONTDEVILA, Clara; ZANCAJO, Adrián. Multiple paths towards education privatization in a globalizing world: a cultural political economy review. Journal of Education Policy, [s.l.], v. 32, n. 6, p. 757-787, 2017. 
VERGER, Antoni; ZANCAJO, Adrián; FONTDEVILA, Clara. La economía política de la privatización educativa: Políticas, tendencias y trayectorias desde una perspectiva comparada. Revista Colombiana de Educación, Bogotá, v. 70, p. 47-48. 2016.

ZUBIZARRETA, José; PAREDES, Ricardo; ROSENBAUM, Paul. Matching for balance, pairing for heterogeneity in an observational study of the effectiveness of forprofit and not-for-profit high schools in Chile. Annals of Applied Statistics, [s.l.], v. 8, n. 1, p. 204-231. 2014.

Texto recibido en 17/08/2020.

Texto aprobado en 30/09/2020. 